Bull. Austral. Math. Soc.

Vol. $63(2001)$ [151-165]

\title{
LARGE DEVIATION RESULTS FOR A $U$-STATISTICAL SUM WITH PRODUCT KERNEL
}

\author{
Y.V. BOROVSKIKH AND N.C. WEBER
}

Large deviation theorems are proved for non-degenerate $U$-statistical sums of degree $m$ with kernel $h\left(x_{1}, \ldots, x_{m}\right)=x_{1} \cdots x_{m}$ under the Cramér condition and under the Linnik condition. The method of proof uses truncation and the contraction technique.

\section{INTRODUCTION AND THEOREMS}

Let $X_{1}, \cdots, X_{n}$ be independent and identically distributed random variables with distribution function $F$. Assume $E X_{1}=\mu \neq 0$ and $0<\sigma^{2}=E\left(X_{1}-\mu\right)^{2}<\infty$. Consider the $U$-statistic of the form

$$
U_{n}=\left(\begin{array}{c}
n \\
m
\end{array}\right)^{-1} \sum_{1 \leqslant i_{1}<\cdots<i_{m} \leqslant n} X_{i_{1}} \cdots X_{i_{m}} .
$$

According to the Hoeffding decomposition

$$
U_{n}-\mu^{m}=\sum_{c=1}^{m} \frac{m(m-1) \cdots(m-c+1)}{n(n-1) \cdots(n-c+1)} \mu^{m-c} \sum_{1 \leqslant i_{1}<\cdots<i_{c} \leqslant n}\left(X_{i_{1}}-\mu\right) \cdots\left(X_{i_{c}}-\mu\right) .
$$

For $m=1, U_{n}-\mu=n^{-1} \sum_{i=1}^{n}\left(X_{i}-\mu\right)$ is the usual sum of independent and identically distributed random variables. Large deviation results with Cramér series for such sums have been developed in many papers including $[1,4,6,7,8]$, both under the Cramér and Linnik conditions and under violation of these conditions.

Let $F_{n}(x)=P\left(\sqrt{n}\left(U_{n}-\mu^{m}\right) /\left(m \sigma|\mu|^{m-1}\right) \leqslant x\right), x \in R$. By the central limit theorem for non-degenerate $U$-statistics, $F_{n}(x) \rightarrow \Phi(x)$ uniformly in $x$ as $n \rightarrow \infty$, where $\Phi(x)$ denotes the standard normal distribution function. Hence for any fixed $m$ and $x$

$$
\left(1-F_{n}(x)\right) /(1-\Phi(x))=(1+o(1)),
$$

as $n \rightarrow \infty$. We are interested in (2) when $x$ tends to infinity together with $n$ and $x=o(\sqrt{n})$. All existing large deviation results for $U$-statistical sums with $x$ in this range require the kernels to be bounded and so they do not apply here. For our special case we have the following result where the coefficients $\lambda_{k m}$ are defined via equation (18).

Received 15th June, 2000

Copyright Clearance Centre, Inc. Serial-fee code: 0004-9727/01 \$A2.00+0.00. 
TheOREM 1. Let $x=o(\sqrt{n})$ and suppose that the Cramér condition

$$
E \exp \left(a\left|X_{1}\right|\right)<\infty
$$

is satisfied for some $a>0$. Then for any fixed $m \geqslant 1$

$$
\left(1-F_{n}(x)\right) /(1-\Phi(x))=\exp \left\{\frac{x^{3}}{\sqrt{n}} \lambda_{m}\left(\frac{x}{\sqrt{n}}\right)\right\}\left(1+O\left(\frac{1+x}{\sqrt{n}}\right)\right)
$$

with the Cramér series

$$
\lambda_{m}(u)=\sum_{k=0}^{\infty} \lambda_{k m} u^{k}
$$

which converges for $0 \leqslant u \leqslant \varepsilon$ for some $\varepsilon>0$.

Further, for $0<\alpha<1 / 2$, let $s=[4 \alpha /(1-2 \alpha)]$ denote the integer part of $4 \alpha /(1-2 \alpha)$. Introduce the truncated Cramér series

$$
\lambda_{m}^{[s]}(u)=\lambda_{0 m}+\lambda_{1 m} u+\cdots+\lambda_{s m} u^{s} .
$$

THEOREM 2. Let $x=o\left(n^{\alpha}\right)$ and suppose that the Linnik condition

$$
E \exp \left\{a\left|X_{1}\right|^{4 \alpha /(2 \alpha+1)}\right\}<\infty
$$

is satisfied for some $a>0$. Then for any fixed $m \geqslant 1$

$$
\left(1-F_{n}(x)\right) /(1-\Phi(x))=\exp \left\{\frac{x^{3}}{\sqrt{n}} \lambda_{m}^{[s]}\left(\frac{x}{\sqrt{n}}\right)\right\}\left(1+O\left(\frac{1+x}{\sqrt{n}}\right)\right) .
$$

\section{Proofs}

In the following we can suppose that $x \geqslant 1$. In fact, by [2, Theorem 6.3.2]

$$
\sup _{x}\left|F_{n}(x)-\Phi(x)\right| \leqslant 36\left(\sigma^{-3} E\left|X_{1}-\mu\right|^{3}+m \exp \left\{2(\sigma / \mu)^{2}\right\}\right) \frac{1}{\sqrt{n}}
$$

for all $1 \leqslant m \leqslant \sqrt{n}$. Further $1-\Phi(x) \geqslant \pi^{-2}$ for $0 \leqslant x \leqslant 1$. Hence for any fixed $m \geqslant 1$ and for $0 \leqslant x \leqslant 1$

$$
\left(1-F_{n}(x)\right) /(1-\Phi(x))=\exp \left\{\frac{x^{3}}{\sqrt{n}} \lambda_{m}\left(\frac{x}{\sqrt{n}}\right)\right\}\left(1+O\left(\frac{1+x}{\sqrt{n}}\right)\right) .
$$

Proof of Theorem 1: The contraction technique for $U$-statistics of degree 2, proposed in [5], was extended in [3] to $U$-statistics with bounded kernels of any degree. Following their approach we define the operator $T_{t}^{m-1}: g \rightarrow T_{t}^{m-1} g$, by

$$
T_{t}^{m-1} g=\frac{E\left\{\prod_{s=1}^{m-1} e^{t g\left(X_{s}\right)} h\left(X_{1}, \ldots, X_{m-1}, \cdot\right)\right\}}{\left(E e^{t g\left(X_{2}\right)}\right)^{m-1}}, \quad t \in R
$$


for any fixed integer $m=2,3 \ldots$, where $g\left(X_{1}\right)$ satisfies the Cramér condition, that is, $E \exp \left(a\left|g\left(X_{1}\right)\right|\right)<\infty$ for some $a>0$.

The following lemma shows that under the Cramér condition (3), for sufficiently small $t$, the equation $g=T_{t}^{m-1} g$ with kernel

$$
h\left(x_{1}, \ldots, x_{m}\right)=x_{1} \cdots x_{m}
$$

has a unique solution $g_{t}$, that is,

$$
g_{t}=T_{t}^{m-1} g_{t}
$$

LEMMA 1. If the Cramér condition (3) is satisfied then the function

$$
g_{t}(x)=\mu_{m}^{m-1}(t) \cdot x, \quad x \in R
$$

is the solution of (6) for kernel (5), where $\mu_{m}(t)$ is an analytical function in the region $0 \leqslant t \leqslant b$ for some sufficiently small $b>0$.

Proof: If some function $g_{t}(x)$ is the solution of (6) then we can write

$$
g_{t}(x)=\frac{\left(E\left(e^{t g_{t}\left(X_{1}\right)} X_{1}\right)\right)^{m-1}}{\left(E e^{t g_{t}\left(X_{1}\right)}\right)^{m-1}} \cdot x .
$$

Let

$$
\mu_{m}(t)=\frac{E\left(e^{t g_{t}\left(X_{1}\right)} X_{1}\right)}{E e^{t_{2 t}\left(X_{1}\right)}}
$$

Hence, for $\mu_{m}(t)$ we obtain the equation

$$
\mu_{m}(t)=\frac{E\left(e^{t \mu_{m}^{m-1}(t) X_{1}} X_{1}\right)}{E e^{t \mu_{m}^{m-1}(t) X_{1}}} .
$$

Futher we shall prove that this equation has a unique analytical solution for small $t$. To show this let

$$
\xi=t \mu_{m}^{m-1}(t)
$$

Then (7) can be written as

$$
t=\xi \cdot\left(\frac{E e^{\xi X_{1}}}{E\left(X_{1} e^{\xi X_{1}}\right)}\right)^{m-1} .
$$

Under condition (3) the functions $E \exp \left(\xi X_{1}\right), E\left(X_{1} \exp \left(\xi X_{1}\right)\right)$ and $E \exp \left(\xi X_{1}\right) / E\left(X_{1} \exp \left(\xi X_{1}\right)\right)$ are analytic for $|\xi| \leqslant a / 2$ and therefore in (9)

$$
t=\xi \cdot \sum_{k=0}^{\infty} c_{k m} \xi^{k}
$$


where the power series converges for small $|\xi|$. For any sufficiently small $|t|$ the equation (10) has a unique real solution $\xi=\xi(t)$, where

$$
\xi(t)=t \cdot \sum_{k=0}^{\infty} d_{k m} t^{k}
$$

with convergent series for small $|t|$. From (8) and (11) we obtain

$$
\mu_{m}^{m-1}(t)=\sum_{k=0}^{\infty} d_{k m} t^{k}
$$

From (12) we see that for any $m \geqslant 2$

$$
\mu_{m}(t)=\sum_{k=0}^{\infty} \mu_{k m} t^{k}
$$

where the power series converges for sufficiently small $t$. This proves Lemma 1.

Let $\psi(t)=\ln E \exp \left\{\operatorname{tg}_{t}\left(X_{1}\right)\right\}$, where $g_{t}(x)$ is given in Lemma 1 . Define $F_{t}$ by

$$
d F_{t}=e^{t g_{t}-\psi(t)} d F
$$

Let $P_{t}$ denote the probability measure under which $X_{1}, X_{2}, \ldots$ are independent and identically distributed from $F_{t}$ and let $E_{t}$ denote the expectation under $P_{t}$. Hence, in (7)

$$
\mu_{m}(t)=E_{t} X_{1} .
$$

Let $t=t(x / \sqrt{n})$, where $t(x / \sqrt{n})$ is the solution of the equation

$$
\mu_{m}^{m}(t)-\mu^{m}=\frac{x}{\sqrt{n}} m \sigma|\mu|^{m-1}
$$

with $x n^{-1 / 2} \rightarrow 0$. The existence of this solution is guaranteed by (13). In fact,

$$
\mu_{m}^{m}(t)=\mu^{m}+\sum_{k=1}^{\infty} \beta_{k m} t^{k},
$$

where the power series converges for sufficiently small $t$. From (15) we obtain

$$
\sum_{k=1}^{\infty} \beta_{k m} t^{k}=\frac{x}{\sqrt{n}} m \sigma|\mu|^{m-1} .
$$

By the inversion theorem for analytic functions we get

$$
t\left(\frac{x}{\sqrt{n}}\right)=\sum_{k=1}^{\infty} t_{k m}\left(\frac{x}{\sqrt{n}}\right)^{k}
$$


with convergent series for small $x n^{-1 / 2}$.

Further, let $U_{n}(t)$ denote the $U$-statistical sum $U_{n}$, where $X_{1}, \ldots, X_{n}$ are independent and identically distributed with distribution function $F_{z}$. By analogy with (1) and by using Lemma 1 we have the Hoeffding decomposition under $P_{t}$

$$
\begin{aligned}
U_{n}(t) & =\mu_{m}^{m}(t)+\frac{m}{n} \sum_{i=1}^{n}\left(g_{t}\left(X_{i}\right)-\mu_{m}^{m}(t)\right) \\
& +\sum_{==2}^{m} \frac{m(m-1) \cdots(m-c+1)}{n(n-1) \cdots(n-c+1)} \mu_{m}^{m-c}(t) \sum_{1 \leqslant i_{1}<\cdots<i_{c} \leqslant n}\left(X_{i_{1}}-\mu_{m}(t)\right) \cdots\left(X_{i_{c}}-\mu_{m}(t)\right) .
\end{aligned}
$$

Let

$$
\sigma_{1}=\frac{1}{n} \sum_{j=1}^{n}\left(g_{t}\left(X_{j}\right)-\mu_{m}^{m}(t)\right) \quad \text { and } \quad v_{n}=\sum_{c=2}^{m} \lambda_{c}(t) \pi_{\mathrm{c}}
$$

where

and

$$
\lambda_{c}(t)=\frac{(m-1) \cdots(m-c+1)}{(n-1) \cdots(n-c+1)} \cdot n^{c-1} \mu_{m}^{-m_{2}(c-1)}(t)
$$

Then

$$
\pi_{c}=n^{-c} \sum_{1 \leqslant i_{1}<\cdots<i_{c} \leqslant n}\left(g_{t}\left(X_{i_{1}}\right)-\mu_{m}^{m}(t)\right) \cdots\left(g_{t}\left(X_{i_{c}}\right)-\mu_{m}^{m}(t)\right) .
$$

$$
U_{n}(t)=\mu_{m}^{m}(t)+m \sigma_{1}+m v_{n} .
$$

Hence using (15)

$$
U_{n}(t)-\frac{x}{\sqrt{n}} m \sigma|\mu|^{m-1}=m \sigma_{1}+m v_{n}+\mu^{m}
$$

and

$$
\begin{aligned}
P\left(U_{n}\right. & \left.-\mu^{m}>\frac{x}{\sqrt{n}} m \sigma|\mu|^{m-1}\right) \\
& =\int I\left(U_{n}\left(y_{1}, \ldots, y_{n}\right)-\mu^{m}>\frac{x}{\sqrt{n}} m \sigma|\mu|^{m-1}\right) \prod_{i=1}^{n} d F\left(y_{i}\right) \\
& =\int \exp \left\{\sum_{i=1}^{n}\left(\psi(t)-t g_{t}\left(y_{i}\right)\right)\right\} I\left(U_{n}-\mu^{m}>\frac{x}{\sqrt{n}} m \sigma|\mu|^{m-1}\right) \prod_{i=1}^{n} d F_{t}\left(y_{i}\right) \\
& =E_{t}\left[e^{n \psi(t)-n t \mu_{m}^{m}(t)-t n \sigma_{1}} I\left(U_{n}(t)-\frac{x}{\sqrt{n}} m \sigma|\mu|^{m-1}-\mu^{m}>0\right)\right] \\
& =e^{n \downarrow(t)-n t \mu_{m}^{m}(t)} \cdot J_{n},
\end{aligned}
$$

where $J_{n}=E_{t} e^{-t n \sigma_{1}} I\left(\sigma_{1}+v_{n}>0\right)$.

Using (16) and the definitions of $\psi(t)$ and $\mu_{m}(t)$ we have

$$
\begin{aligned}
n \psi(t)-n t \mu_{m}^{m}(t) & =-\frac{x^{2}}{2}+\lambda_{0 m} \frac{x^{3}}{\sqrt{n}}+\lambda_{1 m} \frac{x^{4}}{n}+\ldots \\
& =-\frac{x^{2}}{2}+\frac{x^{3}}{\sqrt{n}} \lambda_{m}\left(\frac{x}{\sqrt{n}}\right) .
\end{aligned}
$$


Next we shall estimate $J_{n}$. Let $\xi_{j}=g_{t}\left(X_{j}\right)-\mu_{m}^{m}(t), j=1, \ldots, n$, and introduce the truncated random variables

$$
\bar{\xi}_{j}=\xi_{j} I\left(\left|\xi_{j}\right| \leqslant x \sqrt{n}\right), \quad j=1, \ldots, n .
$$

Then $J_{n}=J_{n}\left(\xi_{1}, \ldots, \xi_{n}\right)$ and $\bar{J}_{n}=J_{n}\left(\bar{\xi}_{1}, \ldots, \bar{\xi}_{n}\right)=E_{t} e^{-t n \bar{\sigma}_{1}} I\left(\bar{\sigma}_{1}+\bar{v}_{n}>0\right)$.

LEMMA 2. If $x \geqslant 1$ and $x n^{-1 / 2} \rightarrow 0$ as $n \rightarrow \infty$, then

$$
J_{n}=\bar{J}_{n}+e^{x^{2} / 2}(1-\Phi(x)) \cdot O\left(\frac{x}{\sqrt{n}}\right)
$$

Proof: Denote

$$
f\left(\xi_{1}, \ldots, \xi_{n}\right)=e^{-t n \sigma_{1}} I\left(\sigma_{1}+v_{n}>0\right)
$$

Note

$$
\begin{aligned}
J_{n}-\bar{J}_{n}= & E_{t}\left[f\left(\xi_{1}, \ldots, \xi_{n}\right)-f\left(\bar{\xi}_{1}, \ldots, \bar{\xi}_{n}\right)\right] \\
= & \sum_{j=1}^{n} E_{t}\left[f\left(\bar{\xi}_{1}, \ldots, \bar{\xi}_{j-1}, \xi_{j}, \ldots, \xi_{n}\right)-f\left(\bar{\xi}_{1}, \ldots, \bar{\xi}_{j}, \xi_{j+1}, \ldots, \xi_{n}\right)\right] \\
= & \sum_{j=1}^{n} E_{t} I\left(\left|\xi_{j}\right|>x \sqrt{n}\right)\left[f\left(\bar{\xi}_{1}, \ldots, \bar{\xi}_{j-1}, \xi_{j}, \xi_{j+1} \ldots, \xi_{n}\right)\right. \\
& \left.-f\left(\bar{\xi}_{1}, \ldots, \bar{\xi}_{j-1}, 0, \xi_{j+1}, \ldots, \xi_{n}\right)\right]
\end{aligned}
$$

Thus

$$
\left|J_{n}-\bar{J}_{n}\right| \leqslant \sum_{j=1}^{n} E_{t}\left[I\left(\left|\xi_{1}\right|>x \sqrt{n}\right)\left(e^{t\left|\xi_{1}\right|}+1\right)\right]\left(E_{t} e^{t \xi_{2}}\right)^{n-j}\left(E_{t} e^{t \bar{\xi}_{1}}\right)^{j-1}
$$

Since

$$
e^{y}=1+y+\theta y^{2} e^{|y|}, \quad|\theta| \leqslant 1, \quad y \in R
$$

under condition (3), $\quad E_{t} e^{t \xi_{2}} \leqslant \exp \left\{c_{1} x^{2} / n\right\}, \quad E_{t} e^{t \bar{\xi}_{1}} \leqslant \exp \left\{c_{1} x^{2} / n\right\}$, and

$$
\begin{aligned}
E_{t} I\left(\left|\xi_{1}\right|>x \sqrt{n}\right) e^{t\left|\xi_{1}\right|} & \leqslant\left(E_{t} e^{2 t\left|\xi_{1}\right|}\right)^{1 / 2}\left(P_{t}\left(\left|\xi_{1}\right|>x \sqrt{n}\right)\right)^{1 / 2} \\
& \leqslant \exp \left\{c_{1} x^{2} / n\right\}\left(P_{t}\left(\left|\xi_{1}\right|>x \sqrt{n}\right)\right)^{1 / 2} \leqslant c_{2} e^{-c_{3} x \sqrt{n}}
\end{aligned}
$$

for some positive constants $c_{i}>0$, which do not depend on $x$ and $n$. Hence in (19)

$$
\begin{aligned}
\left|J_{n}-\bar{J}_{n}\right| & \leqslant 2 c_{2} n \exp \left\{c_{1} x^{2}-c_{3} x \sqrt{n}\right\} \\
& =e^{x^{2} / 2}(1-\Phi(x)) O\left(\frac{x}{\sqrt{n}}\right)
\end{aligned}
$$


since

$$
e^{x^{2} / 2}(1-\Phi(x))=O\left(\frac{1}{x}\right), x \rightarrow \infty
$$

This proves Lemma 2.

Consider $\bar{J}_{n}$. The second Waring formula gives the following representation for $\bar{\pi}_{c}$ :

$$
\dot{\bar{\pi}}_{c}=\sum_{\left\{i_{s} \geqslant 0 ; i_{1}+2 i_{2}+\cdots+i_{c}=c\right\}} \prod_{s=1}^{c}(-1)^{i_{s}-1}\left(s^{i_{s}} i_{s} !\right)^{-1} \bar{\sigma}_{s}^{i_{s}},
$$

where

$$
\bar{\sigma}_{s}=n^{-s} \sum_{k=1}^{n} \bar{\xi}_{k}^{s}, \quad s=1, \ldots, c .
$$

Next we introduce the truncated random variables

$$
\tilde{\sigma}_{j}=\bar{\sigma}_{j} I\left(\left|\bar{\sigma}_{j}\right| \leqslant \delta\right), \quad j=1, \ldots, m
$$

for some sufficiently small $\delta>0$. In addition, $\tilde{J}_{n}, \tilde{v}_{n}, \tilde{\pi}_{c}$ are obtained from $\bar{J}_{n}, \bar{v}_{n}, \bar{\pi}_{c}$, respectively, by substituting $\tilde{\sigma}_{j}$ for $\bar{\sigma}_{j}$.

LEMMA 3. If $x \geqslant 1$ and $x n^{-1 / 2} \rightarrow 0$, then

$$
\bar{J}_{n}=\tilde{J}_{n}+e^{x^{2} / 2}(1-\Phi(x)) O\left(\frac{x}{\sqrt{n}}\right) \text {. }
$$

ProOF: Denote $\varphi\left(\bar{\sigma}_{1}, \ldots, \bar{\sigma}_{m}\right)=e^{-\operatorname{tr} \bar{\sigma}_{1}} I\left(\bar{\sigma}_{1}+\bar{v}_{n}>0\right)$.

Arguing as in the proof of Lemma 2

$$
\begin{aligned}
& \bar{J}_{n}-\tilde{J}_{n}= E_{t}\left[\varphi\left(\bar{\sigma}_{1}, \ldots, \bar{\sigma}_{m}\right)-\varphi\left(\widetilde{\sigma}_{1}, \ldots, \tilde{\sigma}_{m}\right)\right] \\
&=\sum_{k=1}^{m} E_{t} I\left(\left|\bar{\sigma}_{k}\right|>\delta\right)\left[\varphi\left(\widetilde{\sigma}_{1}, \ldots, \tilde{\sigma}_{k-1}, \bar{\sigma}_{k}, \bar{\sigma}_{k+1}, \ldots, \bar{\sigma}_{m}\right)\right. \\
&\left.\quad-\varphi\left(\widetilde{\sigma}_{1}, \ldots, \bar{\sigma}_{k-1}, 0, \bar{\sigma}_{k+1}, \ldots, \bar{\sigma}_{m}\right)\right]
\end{aligned}
$$

from which we obtain

$$
\begin{aligned}
\left|\bar{J}_{n}-\tilde{J}_{n}\right| & \leqslant 2 \sum_{k=1}^{m} E_{t} I\left(\left|\bar{\sigma}_{k}\right|>\delta\right) e^{t n\left|\bar{\sigma}_{1}\right|} \\
& \leqslant 2 \sum_{k=1}^{m}\left(E_{t} e^{2 t n\left|\bar{\sigma}_{2}\right|}\right)^{1 / 2} \cdot\left(P_{t}\left(\left|\bar{\sigma}_{k}\right|>\delta\right)\right)^{1 / 2} .
\end{aligned}
$$

Here

$$
\begin{aligned}
E_{t} e^{2 t n\left|\bar{\sigma}_{1}\right|} & \leqslant E_{t} e^{2 \operatorname{tn} \bar{\sigma}_{1}}+E_{t} e^{-2 \operatorname{ta} \bar{\sigma}_{1}} \\
& =\left(E_{t} e^{2 t \bar{\varepsilon}_{1}}\right)^{n}+\left(E_{t} e^{-2 t\left|\bar{\xi}_{1}\right|}\right)^{n} \leqslant c_{4} e^{c_{5} x^{2}}
\end{aligned}
$$


and

$$
\begin{aligned}
P_{t}\left(\left|\bar{\sigma}_{k}\right|>\delta\right) & \leqslant e^{-\delta x \sqrt{n}} E_{t} \exp \left\{x \sqrt{n}\left|\bar{\sigma}_{k}\right|\right\} \\
& \leqslant e^{-\delta x \sqrt{n}} E_{t} \exp \left\{x \sqrt{n} \bar{\sigma}_{k}\right\}+e^{-\delta x \sqrt{n}} E_{t} \exp \left\{-x \sqrt{n} \bar{\sigma}_{k}\right\}
\end{aligned}
$$

Now

$$
E_{t} \exp \left\{x \sqrt{n} \bar{\sigma}_{k}\right\}=\left(E_{t} \exp \left\{\frac{x \sqrt{n}}{n^{k}} \bar{\xi}_{1}^{k}\right\}\right)^{n}
$$

and using (20)

$$
E_{t} \exp \left\{\frac{x \sqrt{n}}{n^{k}} \vec{\xi}_{1}^{k}\right\} \leqslant 1+\frac{x \sqrt{n}}{n^{k}} E_{t} \bar{\xi}_{1}^{k}+\frac{x^{2}}{n^{2 k-1}} E_{t}\left(\bar{\xi}_{1}^{2 k} \exp \left\{\frac{x \sqrt{n}}{n^{k}}\left|\vec{\xi}_{1}^{k}\right|\right\}\right) .
$$

For $k=1,\left|E_{t} \bar{\xi}_{1}\right| \leqslant c_{6}(x \sqrt{n})^{-1}$, and

$$
E_{t} \bar{\xi}_{1}^{2} \exp \left\{\frac{x}{\sqrt{n}}\left|\bar{\xi}_{1}\right|\right\} \leqslant\left(E_{t} \bar{\xi}_{1}^{4}\right)^{1 / 2}\left(E_{t} \exp \left\{\frac{2 x}{\sqrt{n}}\left|\bar{\xi}_{1}\right|\right\}\right)^{1 / 2} \leqslant c_{7} .
$$

Hence

$$
E_{t} \exp \left\{x \sqrt{n} \bar{\sigma}_{1}\right\} \leqslant \exp \left\{c_{6}+c_{7} x^{2}\right\} .
$$

Now consider (22) when $k \geqslant 2$. In this case $\left|E_{t} \bar{\xi}_{1}^{k}\right| \leqslant c_{8}$, and

$$
\begin{aligned}
E_{t}\left(\bar{\xi}_{1}^{2 k} \exp \left\{\frac{x \sqrt{n}}{n^{k}}\left|\bar{\xi}_{1}^{k}\right|\right\}\right) & \leqslant\left(E_{t} \bar{\xi}_{1}^{4 k}\right)^{1 / 2}\left(E_{t} \exp \left\{\frac{2 x \sqrt{n}}{n^{k}}\left|\bar{\xi}_{1}^{k}\right|\right\}\right)^{1 / 2} \\
& \leqslant\left(E_{t} \bar{\xi}_{1}^{4 k}\right)^{1 / 2}\left(E_{t} \exp \left\{2\left(\frac{x}{\sqrt{n}}\right)^{k}\left|\bar{\xi}_{1}^{k}\right|\right\}\right)^{1 / 2} \leqslant c_{9} .
\end{aligned}
$$

Hence for $k \geqslant 2$,

$$
E_{t} \exp \left\{x \sqrt{n} \bar{\sigma}_{k}\right\} \leqslant \exp \left\{c_{8} \frac{x}{\sqrt{n}}+c_{9}\left(\frac{x}{n^{k-1}}\right)^{2}\right\}
$$

for some positive constants $c_{i}$. These estimates combined with (21) prove Lemma 3 . Next we shall estimate $\widetilde{J}_{n}$.

LEMMA 4. If $x \geqslant 1$ and $x=o(\sqrt{n}), n \rightarrow \infty$, then

$$
\tilde{J}_{n}=e^{x^{2} / 2}(1-\Phi(x))\left(1+O\left(\frac{x}{\sqrt{n}}\right)\right) \text {. }
$$

Proof: Write

$$
\tilde{\pi}_{c}=\tilde{\sigma}_{1} \cdot \alpha_{c}+\beta_{c}, \quad c \geqslant 2
$$


where

$$
\begin{aligned}
& \alpha_{c}=\sum_{\substack{i_{1}+2 i_{2}+\cdots+c i_{c}=c \\
i_{1} \geqslant 1, i_{s} \geqslant 0, s=2, \cdots, c}}\left(\prod_{s=1}^{c}(-1)^{i_{s}-1}\left(s^{i_{s}} i_{s} !\right)^{-1}\right) \tilde{\sigma}_{1}^{i_{1}-1} \tilde{\sigma}_{2}^{i_{2}} \cdots \tilde{\sigma}_{c}^{i_{c}}, \\
& \beta_{c}=\sum_{2 i_{2}+\cdots+i_{c}=c}(-1)^{c+i_{2}+\cdots+i_{c}}\left(\prod_{s=2}^{c}\left(s^{i_{1}} i_{s} !\right)^{-1}\right) \tilde{\sigma}_{2}^{i_{2}} \cdots \tilde{\sigma}_{c}^{i_{c}} .
\end{aligned}
$$

Then

$$
\tilde{v}_{n}=\tilde{\sigma}_{1} \alpha+\beta
$$

where

$$
\alpha=\sum_{c=2}^{m} \lambda_{c}(t) \alpha_{c} \text { and } \beta=\sum_{c=2}^{m} \lambda_{c}(t) \beta_{c} .
$$

Note that $|\alpha| \leqslant a_{1} \cdot \delta \leqslant 1 / 2$ for some constant $a_{1}>0$ and sufficiently small $\delta$. Further, taking account of $(25)$ we have

$$
\tilde{J}_{n}=E_{t} e^{-t n \tilde{\sigma}_{1}} I\left(\widetilde{\sigma}_{1}+\widetilde{v}_{n}>0\right)=E_{t} e^{-t \tilde{\sigma_{1}}} I\left(\tilde{\sigma}_{1}>-\frac{\beta}{1+\alpha}\right)=\sum_{i=1}^{5} \Delta_{i},
$$

where

$$
\begin{aligned}
& \Delta_{1}=E_{t} e^{-t n \sigma_{1}} I\left(\sigma_{1}>0\right) \text {, } \\
& \Delta_{2}=E_{t} e^{-t n \bar{\sigma}_{1}} I\left(\bar{\sigma}_{1}>0\right)-E_{t} e^{-t n \sigma_{1}} I\left(\sigma_{1}>0\right) \text {, } \\
& \Delta_{3}=E_{t} e^{-t \pi \tilde{\sigma}_{1}} I\left(\widetilde{\sigma}_{1}>0\right)-E_{t} e^{-t \pi \bar{\sigma}_{1}} I\left(\bar{\sigma}_{1}>0\right) \text {, } \\
& \Delta_{4}=E_{t} e^{-\operatorname{tn} \tilde{\sigma}_{1}} I\left(\tilde{\sigma}_{1}>-\frac{\beta}{1+\alpha}\right) I\left(\frac{|\beta|}{1+\alpha} \leqslant \frac{1}{n}\right)-E_{t} e^{-\operatorname{tn} \tilde{\sigma}_{1}} I\left(\tilde{\sigma}_{1}>0\right), \\
& \Delta_{5}=E_{t} e^{-t n \tilde{\sigma}_{1}} I\left(\tilde{\sigma}_{1}>-\frac{\beta}{1+\alpha}\right) I\left(\frac{|\beta|}{1+\alpha}>\frac{1}{n}\right) \text {. }
\end{aligned}
$$

Estimate $\Delta_{1}$. Let $\sigma_{t}^{2}=E_{t}\left(g_{t}\left(X_{1}\right)-\mu_{m}^{m}(t)\right)^{2}, x_{t}=\sqrt{n} t \sigma_{t}$,

$$
S=\frac{1}{\sigma_{t} \sqrt{n}} \sum_{i=1}^{n}\left(g_{t}\left(X_{1}\right)-\mu_{m}^{m}(t)\right), \quad \text { and } \quad \Phi_{n}(y)=P_{t}(S \leqslant y), \quad y \in R \text {. }
$$

Note that by Lemma $1,(15)$ and (16)

$$
\begin{gathered}
\sigma_{t}=\sigma|\mu|^{m-1}+O\left(\frac{x}{\sqrt{n}}\right), \quad t=\frac{x}{\sigma|\mu|^{m-1} \sqrt{n}}\left(1+O\left(\frac{x}{\sqrt{n}}\right)\right), \text { and } \\
x_{t}=x+\frac{x^{2}}{\sqrt{n}} O(1) .
\end{gathered}
$$


By definition

$$
\begin{aligned}
\Delta_{1} & =\int e^{-x_{t} y} I(y>0) d \Phi_{n}(y) \\
& =\int_{0}^{\infty} e^{-x_{t} y} d \Phi(y)+\int_{0}^{\infty} e^{-x_{t} y} d\left(\Phi_{n}(y)-\Phi(y)\right) .
\end{aligned}
$$

Recall $e^{z}=1+z \int_{0}^{1} e^{u z} d u, z \in R$, so using (26) we have

$$
\begin{aligned}
\int_{0}^{\infty} e^{-x_{t} y} d \Phi(y) & =\int_{0}^{\infty} \exp \left\{-x y\left(1+O\left(\frac{x}{\sqrt{n}}\right)\right)\right\} d \Phi(y) \\
& =\int_{0}^{\infty} e^{-x y} d \Phi(y)-\frac{x^{2}}{\sqrt{n}} \int_{0}^{1} \int_{0}^{\infty} O(1) y \exp \left\{-x y-\frac{x^{2}}{\sqrt{n}} y u O(1)\right\} d \Phi(y) d u
\end{aligned}
$$

Here

$$
\int_{0}^{\infty} e^{-x y} d \Phi(y)=e^{x^{2} / 2}(1-\Phi(x))
$$

and

$$
\begin{aligned}
\frac{x^{2}}{\sqrt{n}} \int_{0}^{\infty} y \exp \left\{-x y-\frac{x^{2}}{\sqrt{n}} y u O(1)\right\} d \Phi(y) & \leqslant \frac{x^{2}}{\sqrt{n}} \int_{0}^{\infty} y \exp \left\{-x y-\frac{x^{2}}{\sqrt{n}} y u O(1)\right\} d y \\
& =\frac{x^{2}}{\sqrt{n}}\left(x+\frac{x^{2}}{\sqrt{n}} u O(1)\right)^{-2} \\
& =e^{x^{2} / 2}(1-\Phi(x)) O\left(\frac{x}{\sqrt{n}}\right) .
\end{aligned}
$$

Consequently,

$$
\int_{0}^{\infty} e^{-x_{t} y} d \Phi(y)=e^{x^{2} / 2}(1-\Phi(x))\left(1+O\left(\frac{x}{\sqrt{n}}\right)\right)
$$

Further, in (27)

$$
\int_{0}^{\infty} e^{-x_{t} y} d\left(\Phi_{n}(y)-\Phi(y)\right)=-\left(\Phi_{n}(0)-\Phi(0)\right)+x_{t} \int_{0}^{\infty}\left(\Phi_{n}(y)-\Phi(y)\right) e^{-x_{t} y} d y
$$

By the Berry-Esseen theorem (see, for example, [7])

$$
\sup _{y}\left|\Phi_{n}(y)-\Phi(y)\right| \leqslant \sigma_{t}^{-3} E\left|g_{t}\left(X_{1}\right)-\mu_{m}^{m}(t)\right|^{3} \frac{1}{\sqrt{n}}
$$

Hence,

$$
\begin{aligned}
\left|\int_{0}^{\infty} e^{-x_{t} y} d\left(\Phi_{n}(y)-\Phi(y)\right)\right| & \leqslant 2 \sigma_{t}^{-3} E\left|g_{t}\left(X_{1}\right)-\mu_{m}^{m}(t)\right|^{3} \frac{1}{\sqrt{n}} \\
& =e^{x^{2} / 2}(1-\Phi(x)) O\left(\frac{x}{\sqrt{n}}\right)
\end{aligned}
$$

and so we have

$$
\Delta_{1}=e^{x^{2} / 2}(1-\Phi(x))\left(1+O\left(\frac{x}{\sqrt{n}}\right)\right)
$$


Estimate $\Delta_{2}$. Let $\Psi\left(\xi_{1}, \ldots, \xi_{n}\right)=e^{-t n \sigma_{2}} I\left(\sigma_{1}>0\right)$. Then

$$
\begin{aligned}
& \Delta_{2}= E_{t}\left[\Psi\left(\bar{\xi}_{1}, \ldots, \bar{\xi}_{n}\right)-\Psi\left(\xi_{1}, \ldots, \xi_{n}\right)\right] \\
&=\sum_{j=1}^{n} E_{t} I\left(\left|\xi_{j}\right|>x \sqrt{n}\right)\left[\Psi\left(\bar{\xi}_{1}, \ldots, \bar{\xi}_{j-1}, 0, \xi_{j+1}, \ldots, \xi_{n}\right)\right. \\
&\left.\quad-\Psi\left(\bar{\xi}_{1}, \ldots, \bar{\xi}_{j-1}, \xi_{j}, \xi_{j+1}, \ldots, \xi_{n}\right)\right]
\end{aligned}
$$

and therefore

$$
\left|\Delta_{2}\right| \leqslant 2 \sum_{j=1}^{n} E_{t} I\left(\left|\xi_{j}\right|>x \sqrt{n}\right)=2 n P_{t}\left(\left|\xi_{1}\right|>x \sqrt{n}\right) \leqslant 2 E_{t} \xi_{1}^{4} n^{-1}
$$

Hence,

$$
\Delta_{2}=e^{x^{2} / 2}(1-\Phi(x)) O\left(\frac{x}{\sqrt{n}}\right) .
$$

Estimate $\Delta_{3}$. By definition, $\Delta_{3}=E_{t} I\left(\left|\bar{\sigma}_{1}\right|>\delta\right)\left(1-e^{-\operatorname{ta} \bar{\sigma}_{1}} I\left(\bar{\sigma}_{1}>0\right)\right)$ and so from (21) and (23) we have

$$
\Delta_{3}=e^{x^{2} / 2}(1-\Phi(x)) O\left(\frac{x}{\sqrt{n}}\right) .
$$

Estimate $\Delta_{4}$. Clearly

$$
-E_{t} e^{-\operatorname{tn} \tilde{\sigma}_{1}} I\left(0 \leqslant \tilde{\sigma}_{1} \leqslant \frac{1}{n}\right) \leqslant \Delta_{4} \leqslant E_{t} e^{-\operatorname{tn} \tilde{\sigma}_{1}} I\left(-\frac{1}{n} \leqslant \tilde{\sigma}_{1} \leqslant 0\right)
$$

That is,

$$
\begin{aligned}
\left|\Delta_{4}\right| & \leqslant e^{t} E_{t} I\left(\left|\widetilde{\sigma}_{1}\right| \leqslant n^{-1}\right) \\
& =e^{t}\left[E_{t} I\left(\left|\bar{\sigma}_{1}\right| \leqslant \delta\right) I\left(\left|\widetilde{\sigma}_{1}\right| \leqslant n^{-1}\right)+E_{t} I\left(\left|\bar{\sigma}_{1}\right|>\delta\right) I\left(\left|\widetilde{\sigma}_{1}\right| \leqslant n^{-1}\right)\right] \\
& \leqslant e^{t}\left[P_{t}\left(\left|\bar{\sigma}_{1}\right| \leqslant n^{-1}\right)+P_{t}\left(\left|\bar{\sigma}_{1}\right|>\delta\right)\right] .
\end{aligned}
$$

But

$$
P_{t}\left(\left|\bar{\sigma}_{1}\right|>\delta\right) \leqslant e^{-\delta x \sqrt{n}} E_{t} e^{x \sqrt{n}\left|\bar{\sigma}_{1}\right|}
$$

and so from (21) and (23)

$$
P_{t}\left(\left|\bar{\sigma}_{1}\right|>\delta\right)=e^{x^{2} / 2}(1-\Phi(x)) O\left(\frac{x}{\sqrt{n}}\right)
$$

Further in (28)

$$
P_{t}\left(\left|\bar{\sigma}_{1}\right| \leqslant \frac{1}{n}\right)=P_{t}\left(x_{n} \leqslant \bar{s} \leqslant y_{n}\right)
$$


where

$$
\begin{aligned}
& x_{n}=-\left(1+n E_{t} \bar{\xi}_{1}\right) /\left(\bar{\sigma}_{t} \sqrt{n}\right), \quad y_{n}=\left(1-n E_{t} \bar{\xi}_{1}\right) /\left(\bar{\sigma}_{t} \sqrt{n}\right), \\
& \bar{s}=\sum_{i=1}^{n}\left(\bar{\xi}_{i}-E_{t} \bar{\xi}_{1}\right) /\left(\bar{\sigma}_{t} \sqrt{n}\right) \text {, and } \bar{\sigma}_{t}^{2}=E_{t}\left(\bar{\xi}_{1}-E_{t} \bar{\xi}_{1}\right)^{2} \text {. }
\end{aligned}
$$

Let $\bar{\Phi}_{n}(y)=P(\bar{s} \leqslant y)$. Then

$$
\begin{aligned}
P_{t}\left(\left|\bar{\sigma}_{1}\right| \leqslant \frac{1}{n}\right) & \leqslant 2 \sup _{y}\left|\bar{\Phi}_{n}(y)-\Phi(y)\right|+\Phi\left(y_{n}\right)-\Phi\left(x_{n}\right) \\
& \leqslant 2 E_{t}\left|\bar{\xi}_{1}-E_{t} \bar{\xi}_{1}\right|^{3} /\left(\bar{\sigma}_{t}^{3} \sqrt{n}\right)+2\left(\bar{\sigma}_{t} \sqrt{n}\right)^{-1} \\
& =e^{x^{2} / 2}(1-\Phi(x)) O\left(\frac{x}{\sqrt{n}}\right) .
\end{aligned}
$$

Hence, from (28)-(30) we find

$$
\Delta_{4}=e^{x^{2} / 2}(1-\Phi(x)) O\left(\frac{x}{\sqrt{n}}\right) .
$$

Estrmate $\Delta_{5}$. Since $|\alpha| \leqslant 1 / 2$, then

$$
\Delta_{5} \leqslant E_{t} e^{2 \operatorname{tn}|\beta|} I(2 n|\beta| \geqslant 1) .
$$

By Hölder's inequality

$$
\Delta_{5} \leqslant\left(E_{t} e^{4 \operatorname{tn}|\beta|}\right)^{1 / 2}\left(P_{t}(2 n|\beta| \geqslant 1)\right)^{1 / 2}
$$

Using the inequality

$$
\left|y_{1} \ldots y_{m}\right| \leqslant q_{1}^{-1}\left|y_{1}\right|^{q_{1}}+\cdots+q_{m}^{-1}\left|y_{m}\right|^{q_{m}}, \quad q_{1}^{-1}+\cdots+q_{m}^{-1}=1, \quad y_{i} \in R
$$

we obtain

$$
E_{t} e^{4 t n|\beta|} \leqslant a_{2} \sum_{c=2}^{m} \sum_{2 i_{2}+\cdots+a_{c}=c} E_{t} \exp \left\{a_{3} I \sqrt{n}\left|\widetilde{\sigma}_{2}^{i_{2}} \cdots \tilde{\sigma}_{c}^{i_{c}}\right|\right\}
$$

for some positive constants $a_{i}$. Without loss of generality we can suppose that in (32) all $i_{s} \geqslant 1, s=2, \ldots, m$. Otherwise, if $i_{k}=0$ for some $k \geqslant 2$ then $\widetilde{\sigma}_{k}^{i_{k}}=1$. Hence, in (32), if $\delta<1$,

$$
\begin{aligned}
E_{t} \exp \left\{a_{3} x \sqrt{n}\left|\widetilde{\sigma}_{2}^{i_{2}} \cdots \widetilde{\sigma}_{c}^{i_{c}}\right|\right\} & \leqslant E_{t} \exp \left\{a_{3} x \sqrt{n} \delta^{i_{2}+\cdots+i_{c}-1}\left|\widetilde{\sigma}_{j}\right|\right\} \\
& \leqslant E_{t} \exp \left\{a_{3} x \sqrt{n}\left|\widetilde{\sigma}_{j}\right|\right\}, \quad j=2, \ldots, c
\end{aligned}
$$

and so using (24) we get

$$
E_{t} e^{4 t n|\beta|} \leqslant c_{10}
$$


Further in (31) we have for any integer $l \geqslant 8$

$$
P_{t}(2 n|\beta| \geqslant 1) \leqslant(2 n)^{l} E_{t}|\beta|^{l} \leqslant(2 m)^{l} \sum_{c=2}^{m}\left|\lambda_{c}(t)\right|^{l}\left\{n^{l} E_{t}\left|\beta_{c}\right|^{l}\right\} .
$$

Let $r_{j} \geqslant 1, j=1, \ldots, p$, be the non-zero terms in the solution of the equation $2 i_{2}+\cdots+$ $c i_{c}=c, c \geqslant 2$. Hence $i_{k_{1}}=r_{1}, \ldots, i_{k_{p}}=r_{p}$ for some sequence $k_{1}, \ldots, k_{p}$ with $k_{j} \geqslant 2$. Then in (33)

$$
E_{t}\left|\beta_{c}\right|^{l} \leqslant c_{11} \sum_{\left(r_{1}, \ldots, r_{p}\right)} E_{t}\left(\left|\widetilde{\sigma}_{k_{1}}\right|^{l r_{1}} \cdots\left|\widetilde{\sigma}_{k_{p}}\right|^{l r_{p}}\right) \leqslant c_{11} \sum_{\left(r_{1}, \ldots, r_{p}\right)} \sum_{s=1}^{p} E_{t}\left|\bar{\sigma}_{k_{s}}\right|^{p l r_{s}} .
$$

It is easy to see that

$$
n^{l} E_{t}\left|\bar{\sigma}_{k_{s}}\right|^{p l r_{s}} \leqslant c_{12} n^{-t / 2}, \quad s=1, \ldots, p .
$$

Hence in (33), $P_{t}(2 n|\beta| \geqslant 1) \leqslant c_{13} n^{-1}$. Substituting into (31) gives

$$
\Delta_{5}=e^{x^{2} / 2}(1-\Phi(x)) O\left(\frac{x}{\sqrt{n}}\right)
$$

Combining the bounds for the $\Delta_{i}$ completes the proof of Lemma 4.

Theorem 1 follows from (17), (18) and Lemmas 2,3,4.

ProOF OF THEOREM 2: At first we introduce the truncated random variables

$$
\bar{X}_{j}=X_{j} I\left(\left|X_{j}\right| \leqslant n^{(1 / 2)+\alpha}\right), \quad j=1, \ldots, n
$$

and define the $U$-statistic $\bar{U}_{n}=U_{n}\left(\bar{X}_{1}, \ldots, \bar{X}_{n}\right)$. The proof of Theorem 2 follows from Lemmas 5 and 6 below.

Lemma 5. Assume condition (4) holds. Then for $1 \leqslant x \leqslant o\left(n^{\alpha}\right)$

$$
\begin{aligned}
\mid P\left(U_{n}-\mu^{m}>\frac{x}{\sqrt{n}} m \sigma|\mu|^{m-1}\right) & -P\left(\bar{U}_{n}-\bar{\mu}^{m}>\frac{x}{\sqrt{n}} m \bar{\sigma}|\bar{\mu}|^{m-1}\right) \mid \\
& =\exp \left\{\frac{x^{3}}{\sqrt{n}} \lambda_{m}^{[s]}\left(\frac{x}{\sqrt{n}}\right)\right\}(1-\Phi(x)) O\left(\frac{x}{\sqrt{n}}\right),
\end{aligned}
$$

where $\bar{\mu}=\mu\left(\bar{X}_{1}\right)=E \bar{X}_{1}, \bar{\sigma}^{2}=\sigma^{2}\left(\bar{X}_{1}\right)=E\left(\bar{X}_{1}-\bar{\mu}\right)^{2}$.

Proof: By analogy with the proof of Lemma 2 we can write the inequality

$$
\left|P\left(U_{n}-\mu^{m}>\frac{x}{\sqrt{n}} m \sigma|\mu|^{m-1}\right)-P\left(\bar{U}_{n}-\bar{\mu}^{m}>\frac{x}{\sqrt{n}} m \bar{\sigma}|\bar{\mu}|^{m-1}\right)\right| \leqslant 2 n P\left(\left|X_{1}\right|>n^{(1 / 2)+\alpha}\right) .
$$

Here

$$
P\left(\left|X_{1}\right|>n^{(1 / 2)+\alpha}\right) \leqslant e^{-a n^{2 \alpha}} E \exp \left\{a\left|X_{1}\right|^{4 \alpha /(2 \alpha+1)}\right\} .
$$


Since $x \geqslant 1$ and $x n^{-\alpha} \rightarrow 0$ as $n \rightarrow \infty$ then

$$
\begin{aligned}
e^{-a n^{2 \alpha}}= & e^{-(\alpha / 2) n^{2 \alpha}} \exp \left\{\frac{x^{3}}{\sqrt{n}} \lambda_{m}^{[s]}\left(\frac{x}{\sqrt{n}}\right)-\frac{x^{2}}{2}\right\} \\
& \quad \exp \left\{-n^{2 \alpha}\left[\frac{a}{2}-\left(\frac{x}{n^{\alpha}}\right)^{2}\left(\frac{1}{2}-\frac{x}{\sqrt{n}} \lambda_{m}^{[s]}\left(\frac{x}{\sqrt{n}}\right)\right)\right]\right\} \\
= & e^{-(\alpha / 2) n^{2 \alpha}} \exp \left\{\frac{x^{3}}{\sqrt{n}} \lambda_{m}^{[s]}\left(\frac{x}{\sqrt{n}}\right)\right\}(1-\Phi(x)) O(1),
\end{aligned}
$$

completing the proof of Lemma 5.

LEMMA 6. If $1 \leqslant x \leqslant o\left(n^{\alpha}\right)$ and condition (4) is satisfied then

$$
P\left(\bar{U}_{n}-\bar{\mu}^{m}>\frac{x}{\sqrt{n}} m \bar{\sigma}|\bar{\mu}|^{m-1}\right)=\exp \left\{\frac{x^{3}}{\sqrt{n}} \lambda_{m}^{[s]}\left(\frac{x}{\sqrt{n}}\right)\right\}(1-\Phi(x))\left(1+O\left(\frac{x}{\sqrt{n}}\right)\right) .
$$

Proof: We shall apply Theorem 1 . In the following $\bar{\mu}, \bar{\sigma}, \bar{t}, \bar{\psi}, \ldots$ denote $\mu, \sigma, t, \psi, \ldots$ respectively, where, instead of $X_{1}$, we substitute $\bar{X}_{1}$. For example, $\bar{\mu}=E \bar{X}_{1}$ and $\bar{\sigma}^{2}=E\left(\bar{X}_{1}-\bar{\mu}\right)^{2}$. At first we note that for $|z| \leqslant(1 / 2) a n^{-(1 / 2)+\alpha}$,

$$
\begin{gathered}
E \exp \left(z \bar{X}_{1}\right) \leqslant E \exp \left\{a\left|X_{1}\right|^{4 \alpha /(2 \alpha+1)}\right\}, \\
\left|E \exp \left(z \bar{X}_{1}\right) \bar{X}_{1}\right|^{2} \leqslant E X_{1}^{2} E \exp \left\{a\left|X_{1}\right|^{4 \alpha /(2 \alpha+1)}\right\} .
\end{gathered}
$$

Hence, given condition (4), the function $E \exp \left(z \bar{X}_{1}\right) / E \exp \left(z \bar{X}_{1}\right) \bar{X}_{1}$ in (9) is majorised by an analytic function uniformly in $n$ for $|z| \leqslant(1 / 2) a n^{-(1 / 2)+\alpha}$. Further, by analogy with (18)

$$
n \bar{\psi}(\bar{t})-n \bar{t} \bar{\mu}_{m}^{m}(\bar{t})=-\frac{x^{2}}{2}+\frac{x^{3}}{\sqrt{n}} \bar{\lambda}_{m}\left(\frac{x}{\sqrt{n}}\right)
$$

Therefore, under condition (4), applying Theorem 1 for $1 \leqslant x \leqslant o\left(n^{\alpha}\right)$

$$
P\left(\bar{U}_{n}-\bar{\mu}^{m}>\frac{x}{\sqrt{n}} m \bar{\sigma}|\bar{\mu}|^{m-1}\right)=(1-\Phi(x)) \exp \left\{\frac{x^{3}}{\sqrt{n}} \bar{\lambda}_{m}\left(\frac{x}{\sqrt{n}}\right)\right\}\left(1+O\left(\frac{x}{\sqrt{n}}\right)\right),
$$

with Cramér series

$$
\bar{\lambda}_{m}(u)=\sum_{k=0}^{\infty} \bar{\lambda}_{k m} u^{k}
$$

which converges for $0 \leqslant u \leqslant \varepsilon n^{-(1 / 2)+a}$ and sufficiently small $\varepsilon>0$. The coefficients $\bar{\lambda}_{k m}=\lambda_{k m}\left(\bar{X}_{1}\right)$ depend on the moments of the truncated random variable $\bar{X}_{1}$.

Let

$$
\bar{\lambda}_{m}(u)=\sum_{k=0}^{s} \bar{\lambda}_{k m} u^{k}+\rho(u)
$$


where $\rho(u)=\sum_{k=s+1}^{\infty} \bar{\lambda}_{k m} u^{k}$. Note $s+1 \geqslant 4 \alpha /(1-2 \alpha)$ and in (34) the series is convergent and uniformly bounded. Hence for $x=o\left(n^{\alpha}\right)$

$$
\frac{x^{3}}{\sqrt{n}} \rho\left(\frac{x}{\sqrt{n}}\right)=O(1) \frac{x^{3}}{\sqrt{n}}\left(\frac{x}{\sqrt{n}}\right)^{s+1}=O\left(\frac{x}{\sqrt{n}}\right) .
$$

Moreover

$$
\sum_{k=0}^{s} \bar{\lambda}_{k m}\left(\frac{x}{\sqrt{n}}\right)^{k}=\lambda_{m}^{[s]}\left(\frac{x}{\sqrt{n}}\right)+O\left(\frac{1}{n}\right)
$$

Thus

$$
\exp \left\{\frac{x^{3}}{\sqrt{n}} \bar{\lambda}_{m}\left(\frac{x}{\sqrt{n}}\right)\right\}=\exp \left\{\frac{x^{3}}{\sqrt{n}} \lambda_{m}^{[s]}\left(\frac{x}{\sqrt{n}}\right)\right\}\left(1+O\left(\frac{x}{\sqrt{n}}\right)\right)
$$

This proves Lemma 6 .

\section{REFERENCES}

[1] H. Cramér, 'Sur un nouveaux théorème limit de la théorie des probabilités', Actualités Sci. Indust. 736 (1938), 5-23.

[2] Y.V. Borovskikh, $U$-statistics in Banach Spaces (VSP, Utrecht, The Netherlands, 1996).

[3] Y.V. Borovskikh and N.C. Weber, On large deviations of $U$-statistics, Technical Report 00-4 (2000) (School of Mathematics and Statistics, University of Sydney, Australia, 2000).

[4] I.A. Ibragimov and Y.V. Linnik, Independent and stationary sequences of random variables (Wolters-Noordhoff Publishing, Groningen, The Netherlands, 1971).

[5] R.W. Keener, J. Robinson and N.C. Weber, 'Tail probability approximations for U-statistics', Statist. Probab. Lett. 37 (1998), 59-65.

[6] Y.V. Linnik, The collected works. Probability theory (Nauka Publishers, Leningrad, 1981).

[7] V.V. Petrov, Sums of independent random variables (Springer-Verlag, Berlin, Heidelberg, New York, 1975).

[8] L. Saulis and V. Statulevicius, Limit theorems on large deviations (Mokslas Publishers, Vilnius, 1989).

Department of Applied Mathematics

Transport University 190031, St. Petersburg

Russia

e-mail: borovski@spb.cityline.ru
School of Mathematics and Statistics, F07

University of Sydney

New South Wales 2006

Australia

e-mail: neville@maths.usyd.edu.au 